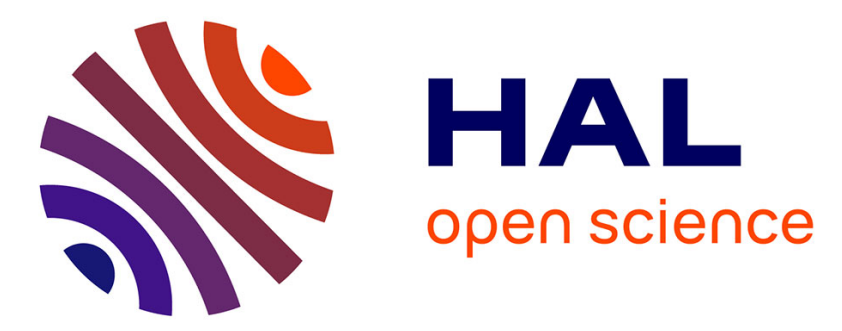

\title{
The interaction of helical tip and root vortices in a wind turbine wake
}

Michael Sherry, Andras Nemes, David Lo Jacono, Hugh M. Blackburn, John Sheridan

\section{> To cite this version:}

Michael Sherry, Andras Nemes, David Lo Jacono, Hugh M. Blackburn, John Sheridan. The interaction of helical tip and root vortices in a wind turbine wake. Physics of Fluids, 2013, vol. 25, pp.1-16. 10.1063/1.4824734. hal-00913622

\section{HAL Id: hal-00913622 \\ https://hal.science/hal-00913622}

Submitted on 4 Dec 2013

HAL is a multi-disciplinary open access archive for the deposit and dissemination of scientific research documents, whether they are published or not. The documents may come from teaching and research institutions in France or abroad, or from public or private research centers.
L'archive ouverte pluridisciplinaire HAL, est destinée au dépôt et à la diffusion de documents scientifiques de niveau recherche, publiés ou non, émanant des établissements d'enseignement et de recherche français ou étrangers, des laboratoires publics ou privés. 


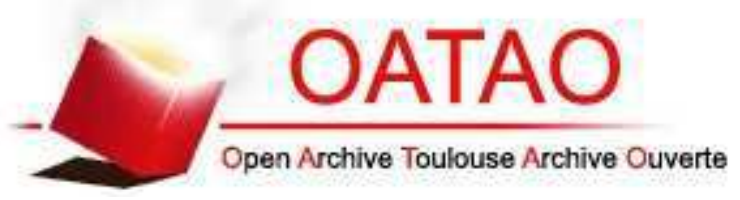

\section{Open Archive TOULOUSE Archive Ouverte (OATAO)}

OATAO is an open access repository that collects the work of Toulouse researchers and makes it freely available over the web where possible.

This is an author-deposited version published in : http://oatao.univ-toulouse.fr/ Eprints ID : 10414

To link to this article : DOI:10.1063/1.4824734

URL : http://dx.doi.org/10.1063/1.4824734

\section{To cite this version :}

Sherry, Michael and Nemes, Andras and Lo Jacono, David and

Blackburn, Hugh M. and Sheridan, John The interaction of helical tip and root vortices in a wind turbine wake. (2013) Physics of Fluids, vol. 25 . pp. 1-16. ISSN 1070-6631

Any correspondance concerning this service should be sent to the repository administrator: staff-oatao@ listes-diff.inp-toulouse.fr 


\title{
The interaction of helical tip and root vortices in a wind turbine wake
}

\author{
Michael Sherry, ,a) András Nemes, ${ }^{1}$ David Lo Jacono, ${ }^{1,2}$ \\ Hugh M. Blackburn, ${ }^{1}$ and John Sheridan ${ }^{1}$ \\ ${ }^{1}$ Department of Mechanical and Aerospace Engineering, Monash University, \\ Melbourne, Vic 3800, Australia \\ ${ }^{2}$ Institut de Mécanique des Fluides de Toulouse (IMFT), CNRS, UPS, Université de Toulouse, \\ Allée Camille Soula, F-31400 Toulouse, France
}

\begin{abstract}
Analysis of the helical vortices measured behind a model wind turbine in a water channel are reported. Phase-locked measurements using planar particle image velocimetry are taken behind a Glauert rotor to investigate the evolution and breakdown of the helical vortex structures. Existing linear stability theory predicts helical vortex filaments to be susceptible to three unstable modes. The current work presents tip and root vortex evolution in the wake for varying tip speed ratio and shows a breaking of the helical symmetry and merging of the vortices due to mutual inductance between the vortical filaments. The merging of the vortices is shown to be steady with rotor phase, however, small-scale non-periodic meander of the vortex positions is also observed. The generation of the helical wake is demonstrated to be closely coupled with the blade aerodynamics, strongly influencing the vortex properties which are shown to agree with theoretical predictions of the circulation shed into the wake by the blades. The mutual inductance of the helices is shown to occur at the same non-dimensional wake distance.
\end{abstract}

\section{INTRODUCTION}

Rotor systems such as wind turbines, airplane propellers, and helicopter rotors generate complex wakes containing helical vortex structures. In the case of three-bladed horizontal-axis wind turbines (HAWTs), vortices form in the tip and root region in the wake of each blade, resulting in three counter-rotating pairs of helical vortices. The evolution and breakdown dynamics of these helical vortices is not fully understood and remains an important question in wind energy, ${ }^{1-5}$ aviation, ${ }^{6,7}$ and marine industries, ${ }^{8}$ and is also of fundamental interest in vortex dynamics. ${ }^{9-14}$ In the case of helicopter rotors, these coherent vortex structures influence flight aerodynamics, ${ }^{6}$ generate noise, ${ }^{15}$ and result in the highly undesirable vortex ring state. ${ }^{7}$ For wind turbines, these helical vortices play an important role in the development of the wake directly downstream of the rotor (the near-wake) and their evolution and breakdown dynamics influence the characteristics of the resultant far-wake many rotor diameter downstream. This has direct consequences in wind farms where wake interaction with downstream turbines may introduce dynamic blade loading and lead to a drop in wind farm performance. ${ }^{16}$ Such challenges in industry provide the motivation to investigate these structures with an aim to include their dynamics in wake models.

Fundamental studies on helical vortices have shown that their symmetrical helical structure is unstable to small perturbations. Widnall ${ }^{10}$ showed that a single helical filament in an inviscid fluid is linearly unstable to three types of perturbation modes: a short-wave and a long-wave mode along the filament, and a mutual induction mode where subsequent turns of the filament interact. Gupta and

\footnotetext{
a)Electronic mail: msherry@ucalgary.ca. Present address: Department of Mechanical Engineering, University of Calgary, Calgary, Alberta T2N 1N4, Canada.
} 
Loewy ${ }^{11}$ extended this to consider multiple vortical filaments and Hattori and Fukumoto ${ }^{17}$ included consideration of small wavelengths in the analysis. Theoretical investigations of idealized rotor wake models have led to more complex analytical configurations of multiple tip vortex filaments, which have been investigated with ${ }^{12,14}$ and without ${ }^{13}$ a hub vortex. The hub vortex is modeled in the Joukowsky wake configuration: as a single columnar vortex of opposite sign and circulation strength to the sum of the tip vortices, aligned along the axis of the rotor.

In the aforementioned analytical investigations, the inviscid models of helical vortex systems which include the hub vortex were found to be inherently unstable. Nevertheless, experimental

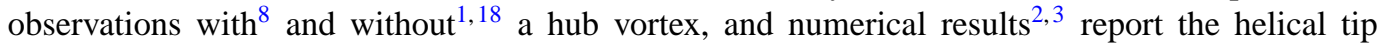
vortices persisting far into the wake. The distance to breakdown of the wake varies between studies, as wake properties are dependent on a large number of operational parameters. Currently, there is no universal model able to predict experimental observations of wake breakdown.

In terms of modeling the helical wake structure, there are two idealized systems that have been proposed: ${ }^{19}$ the Joukowsky wake model based on a rotating horseshoe vortex, and the Betz wake model composed of rigid helical vortex sheets generated by the blades. Okulov and Sørensen ${ }^{14}$ proposed that modeling of rotor wakes for stability analysis requires a relaxation of the ideal constant circulation (i.e., Joukowsky) blade assumption in order to capture sufficient physics. Further, the authors proposed that the blade wake, a remnant of the blade boundary layer and trailing vorticity sheet, should be included due to the role it plays in the vortex interactions of the system. Their subsequent stability analysis of helical vortices embedded in various assigned rotational flow fields demonstrated that the helix stability conditions were strongly dependent on the assigned flow field. This also supports Okulov and Sørensen's ${ }^{14}$ hypothesis that the tip and hub vortex interaction stabilizes an otherwise inherently unstable system.

Experimental flow visualizations ${ }^{1}$ and numerical simulations ${ }^{3}$ have all shown that the tip vortices of a wind turbine undergo pairing, with a resulting entanglement and merging of vortices. A similar phenomenon occurs in other helical vortex systems ${ }^{6-8}$ and is a direct result of the Biot-Savart law. Recent flow visualizations of a marine propeller by Felli, Camussi, and Felice ${ }^{8}$ provided further insight, showing that the filament pairing for a three-bladed rotor occurs in a two-step process, with two filaments initially entangling, followed by their interaction with the remaining filament. This observation differs from the results shown in the numerical simulations of a wind turbine wake by Ivanell $e t \mathrm{al}^{3}{ }^{3}$ The tip vortices in their three-bladed rotor configuration interact with their neighbouring vortices in an alternating pattern downstream, suggesting that the helices are undergoing a single grouping process. These two observations could be the result of differing patterns present in the pairing of tip vortices in rotor wakes. Although in the above studies the wake symmetry is analogous,${ }^{20}$ the two systems are not identical: the vortex wake of a marine propeller contains a single rectilinear hub vortex irrespective of the number of blades, $B$ (i.e., $B+1$ vortices), whereas a coherent root vortex forms behind each wind turbine blade (i.e., $2 B$ vortices). Felli, Camussi, and Felice's ${ }^{8}$ results indicate that the tip vortex of the marine propeller becomes unstable earlier than the hub vortex. Ivanell et al. ${ }^{3}$ did not focus on evolution of the root vortices. In fact, helical root vortices have received little attention in HAWT studies. ${ }^{21}$ As such, there is a clear need for further experiments in order to identify the source of discrepancies between current numerical and experimental results, and the influence of the root vortex on the tip vortex evolution.

For experimental investigations on rotor wakes, small-scale experiments are preferable over large-scale as they provide both a wide field of view (FOV) and the high resolution data needed to resolve the induced velocities of multiple vortices. A drawback of small-scale experimental models is the reduced aerodynamic performance of the rotor airfoils. Geometric down-scaling of HAWT blades has been shown to result in poor dynamic scaling and a wake unsuitable for wake stability studies, ${ }^{21}$ reaffirming that the near-wake dynamics are coupled strongly to the blade aerodynamics, and that investigation of the former requires knowledge of the latter. Experimental studies must now focus on designing appropriate rotors for helical wake studies in order to generate wake profiles close to those of idealized wake models. ${ }^{22}$

The current paper investigates the wake of a three-bladed HAWT rotor, with a blade design based on the Glauert ${ }^{23}$ optimum rotor. The aim is to generate a wake that permits investigation of the generation, evolution, and breakdown of the helical structures. A relationship between these helical 
structures and the governing parameters of the system is sought. Section II provides a description of the method and experimental setup. The measurements and analysis are provided in Sec. III and are followed by conclusions.

\section{DESIGN AND EXPERIMENTAL SETUP}

\section{A. Glauert optimum rotor design}

The blades of the experimental rotor were based on Glauert's optimum rotor. ${ }^{23}$ The optimum rotor design maximizes the power coefficient, $C_{P}$, based on inputs of blade number, airfoil profile, tip speed ratio, $\lambda$, and blade chord Reynolds number, $R e_{c}$. The tip speed ratio is the ratio between the rotational speed at the tip, $\Omega R$, and the freestream velocity, $U_{\infty}$. It is the dominant parameter in wake stability studies, controlling the pitch of the helical filament, $h$. The Reynolds number is defined by the ratio of the product of relative velocity, $U_{\text {rel }}(R)=\sqrt{U_{\infty}^{2}+\left(\lambda U_{\infty}\right)^{2}}$ and chord length at the blade tip, $c(R)$, to the fluid kinematic viscosity, $v$.

The rotor was designed with three blades, typical of full scale HAWTs. The rotor has a radius of $R=115 \mathrm{~mm}$, with the length chosen to optimize blockage considerations (see Sec. II B). A NACA4412 profile was selected for the blade design, based on the availability of tabulated airfoil data $^{24}$ and prior work ${ }^{21}$ evaluating the low Reynolds number performance of the cross-section. The design angle of attack was set to $\alpha=6^{\circ}$. This choice diverged from the Glauert optimum rotor design of maximum $C_{l} / C_{d}$ at $\alpha=7.3^{\circ}$, to compensate for the lower operating Reynolds number, $R e_{c} \approx 11000$ compared to the tabulated airfoil data. This decrease in angle of attack was to keep the airfoil's operating angle in the lift curve's linear region along the entire span of the lifting surface. An empirical tip speed ratio formula ${ }^{25}$ determined the optimized design tip speed ratio of $\lambda_{d}=3.5$. The Glauert optimal blade design was further modified in the tip region by increasing the tip chord, as prior research ${ }^{26,27}$ has shown that the tip vortex core size is approximately $10 \%$ of the chord. This resulted in the slight kink in the tip region. Finally, a high degree of twist was incorporated to avoid flow separation on the airfoil in the root region. The design conditions therefore follow the Glauert optimum rotor design with a conservative choice of angle of attack to avoid stall. The resulting Glauert rotor blade's twist, $\phi$, and taper are presented in Figure 1. The blade's lifting surface terminates prior to the rotational axis (at $0.245 R$ ) in a similar fashion to full-scale HAWTs, with an experimental goal to generate distinct coherent helical root vortices predicted by theory.

As the focus of the investigation is the dynamics of the vortex wake, the key parameter of interest in the blade design is the blade bound circulation distribution, $\Gamma_{B}(r)$. From Helmholtz's vortex theorems, a change in the blade bound circulation along the blade results in a vortex of equal strength being shed into the wake at that blade location. Figure 2 shows the blade bound circulation,

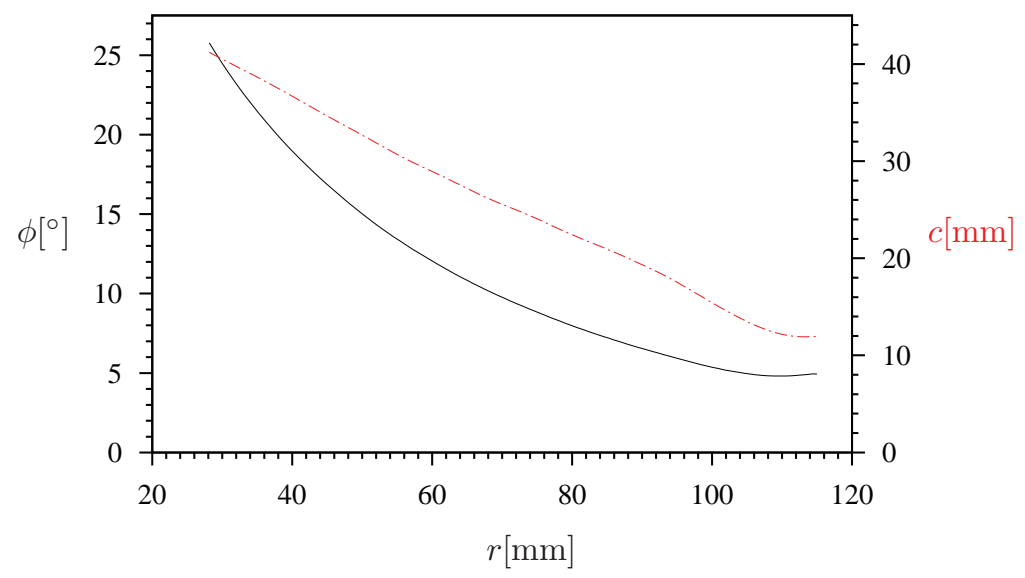

FIG. 1. Spanwise twist, $\phi$ (solid line, black) and chord, $c$ (dashed line, red) variation of the Glauert optimum rotor design. 


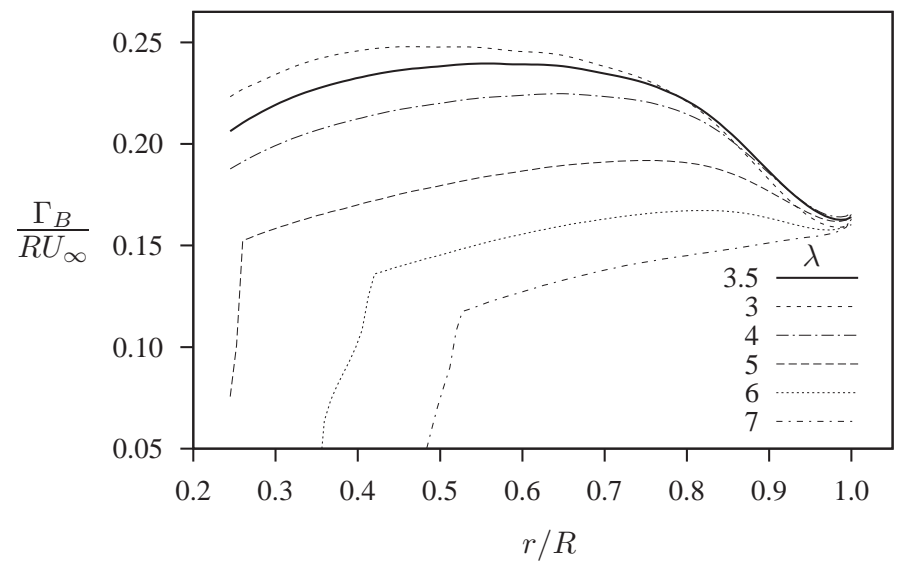

FIG. 2. Blade element momentum prediction of the bound circulation distribution, $\Gamma_{B}(r)$, for the NACA 4412 blade and the various tip speed ratios, $\lambda$, investigated.

$\Gamma_{B}(r)$, predicted by the blade element momentum (BEM) method with tip loss correction of Shen et al. ${ }^{28}$ over a range of tip speed ratio $(3 \leq \lambda \leq 7)$.

The BEM method predicts a smaller bound circulation with larger tip speed ratio, due to the reducing angle of attack. This corresponds to a reduction in the generated lift force. The lowest tip speed ratio, $\lambda=3$, predicts a higher performance of the blade than $\lambda_{d}$, although at the experimental Reynolds number, the $\lambda=3$ case causes the blade angle of attack to be operating in the pre-stall region. The circulation predictions near the tip have approximately the same value for all $\lambda$ due to the rotor operating in the linear regime of airfoil performance. A zero circulation condition physically exists at the tip, $r / R=1$, however the 2D BEM method employed does not enforce this condition and is not shown in Figure 2. However, the spanwise variation in the bound circulation results in additional vorticity shed into the blade wake. Therefore, while the vorticity shed at the tip has approximately the same predicted strength at all $\lambda$, additional vorticity shed into the blade wake is entrained into the tip vortices in the vortex roll-up process. This results in fully formed tip vortices of varying strength for different tip speed ratios. From Figure 2, the $\lambda=3$ tip vortex is predicted to be strongest, with decreasing strength as the tip speed ratio is increased. For off design values of tip speed ratio, $\lambda>4$, the root sections of the blade begin to experience negative angles of attack. At this point, there is a significant drop in performance and the BEM method does not converge as the lift loading approaches zero. The location of maximum positive slope of $\Gamma_{B}(r)$ is also displaced radially outward with increasing $\lambda$, predicting root vortex formation further away from the turbine nacelle. These predictions are compared to wake measurements in Sec. III.

\section{B. Experimental setup}

The experiments were conducted in the FLAIR free-surface recirculating water channel. ${ }^{21,29-32}$ The water channel has a test section of $4000 \mathrm{~mm}$ in length, $600 \mathrm{~mm}$ in width, and $800 \mathrm{~mm}$ in depth, giving the rotor model blockage at $9 \%$, based on swept area. The freestream velocity was maintained at $U_{\infty}=200 \mathrm{~mm} / \mathrm{s}$ for all measurements and the water temperature varied by less than $T=0.1^{\circ} \mathrm{C}$ during acquisition. The experimental setup is shown in Figure 3.

The turbine was driven at a constant tip speed ratio by a micro-stepping drive (OEM350-650 and 6K2 Controller, Parker). The drive shaft of the motor was situated above the water channel and a timing belt transferred the torque to the model turbine shaft through the tower support. An optical encoder (HBM5-1250, US Digital) attached to the drive shaft recorded the turbine rotational speed and blade position. The rotational speed was varied to allow investigation of the tip speed ratio range, $3 \leq \lambda \leq 7$.

Particle image velocimetry (PIV) was used to reconstruct two-dimensional (2D) velocity fields behind the turbine. The flow was seeded with hollow microspheres (Sphericel 110P8, Potters 


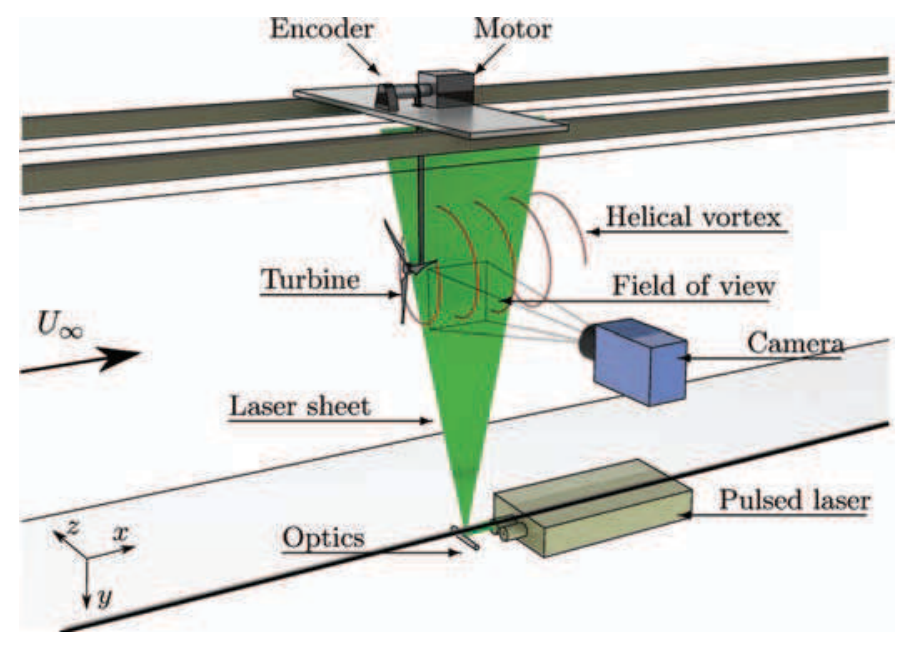

FIG. 3. Schematic of the rig showing the experimental configuration. The simulated path of a single tip vortex is shown as it intersects the (xy)-measurement plane illuminated by the laser. The measurement plane is aligned with the rotational axis of the turbine.

Industries, Inc.) with a nominal diameter of $13 \mu \mathrm{m}$ and a specific weight of $1.1 \mathrm{~g} \mathrm{~cm}^{-3}$. Two Nd:YAG pulsed lasers (Minilite II Q-Switched lasers, Continuum) were directed through optics to produce two $2 \mathrm{~mm}$ thick coplanar sheets pulsed at an interval of $\Delta t$ apart, illuminating the particles in a vertical-streamwise plane on the wake center-line, see Figure 3. A CCD camera (pco.4000, PCO AG) with double shutter, $4008 \times 2672$ pixel resolution, and equipped with a $105 \mathrm{~mm}$ lens (AF105 Nikkor, Nikon Corporation) captured image pairs of the illuminated plane. In-house cross-correlation software ${ }^{33}$ calculated the instantaneous velocity field $(u, v)$ from the image pairs using multi-pass windows down to $32 \times 32$ pixel interrogation windows with $75 \%$ overlap to give $498 \times 331$ vectors with a spatial resolution of $4.3 \times 10^{-3} R$. Where appropriate, the velocity, vorticity, and length scales are made dimensionless by $U_{\infty}$, and $R$. Several post-processing techniques were used, and are discussed in the results where relevant.

PIV measurements were taken on the center plane of the turbine wake, with a region of interest in the half of the wake that did not include the tower support geometry. A three axis traverse (IMC-S8 controller, ISEL) was used to position the camera at multiple locations to capture the wake evolution while maintaining the high spatial resolution of the vector fields. Two sets of wake measurements were performed to examine the vortex dynamics in the wake. The first set of acquisition, denoted as M1, identified the behavior of the tip or root vortices in the wake with varying $\lambda$, and is discussed in Sec. III A. The second set of acquisition, denoted as M2, recorded overall wake development for two $\lambda$, and is discussed in Sec. III B.

\section{RESULTS}

\section{A. Spatial evolution of helical vortices}

To capture the spatial evolution of the helical tip and root vortices in the wake, the M1 measurements captured phase-locked PIV frames at multiple blade angles. For each tip speed ratio $(\lambda=3,4$, $5,6,7$ ), measurements were recorded at 4 camera positions ( 3 streamwise positions for the tip, and 1 for the root vortices) and at 36 blade angles of $10^{\circ}$ increments. An angle of $\theta=0^{\circ}$ represents a blade aligned with the measurement plane. The tip vortices were captured up to a streamwise distance of $x / R=5$ whereas the root vortices were captured up to $x / R=2$ due to rapid root vortex breakdown. PIV snapshots were acquired at each revolution, with an ensemble average of 25 snapshots at each phase. This number of snapshots was found to accurately capture the spatial location of the vortices. The field of view in M1 was restricted to the shear layers at $r_{F} \approx 1 R\left(r_{F} \approx 0.2-0.4 R\right)$ containing 

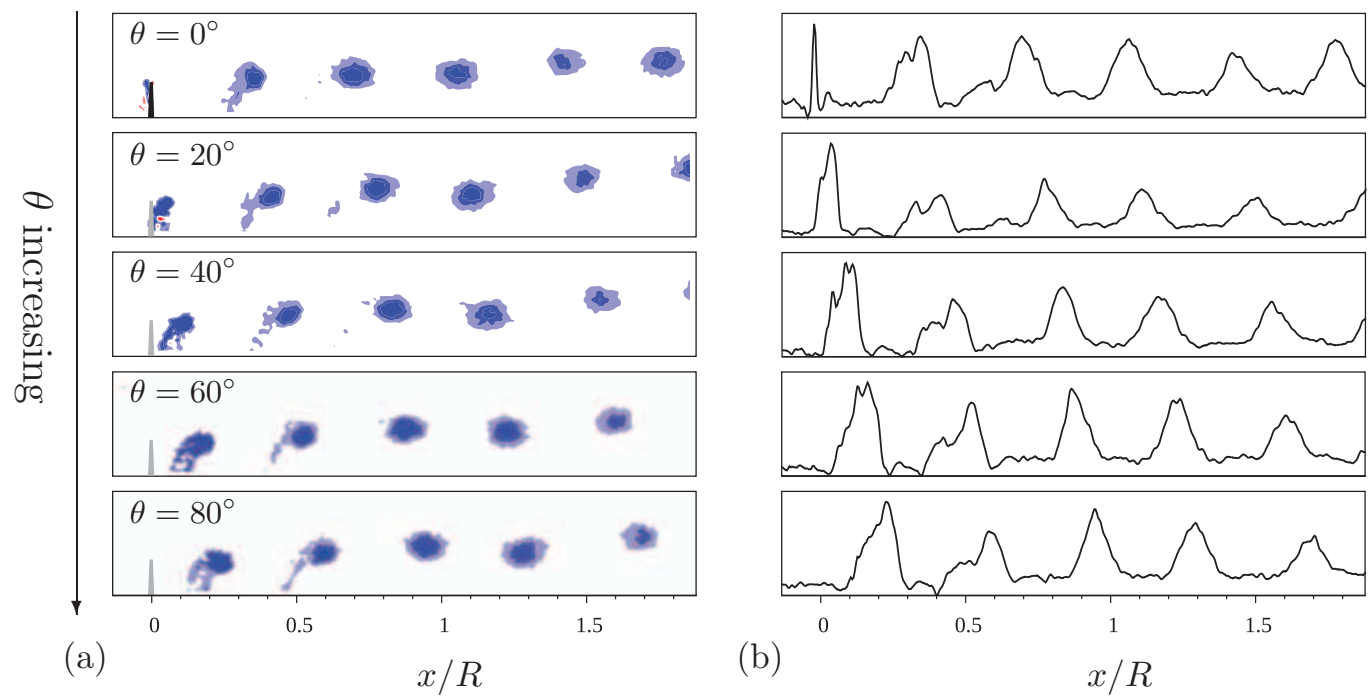

(b)

$x / R$

FIG. 4. (a) Out-of-plane vorticity contours of the $2 \mathrm{D}$ field, $\bar{\omega}_{z}$, in the tip vortex region for $\lambda=5$. Evolution of the vortices can be seen with increasing blade-angle, $\theta$. (b) The corresponding $1 \mathrm{D}$ signal of vorticity intensity, $I_{\bar{\omega}_{z}}(x / R)$, used to map the streamwise location of vortices against blade angle in Figure 5.

the coherent tip (root) vortices. The vorticity fields reveal the evolution of the tip and root vortices with blade angle in the wake, an example of the tip vortices is shown in Figure 4(a) for $\lambda=5$.

The streamwise evolution of the tip and root vortices for $3 \leq \lambda \leq 7$ are shown in the space-phase vorticity intensity maps in Figure 5 (left and right figures, respectively). The contours represent the intensity, $I_{\bar{\omega}_{z}}(x)$, of the averaged phase-locked out-of-plane vorticity. The intensity is the average vorticity in the vertical (radial direction in the helical coordinate system) direction at each streamwise location,

$$
I_{\bar{\omega}_{z}}(x)=\sum_{y} \bar{\omega}_{z}(x, y)
$$

within a PIV frame with the FOV limited to the vortex shear layer (as shown in Figure 4).

In Figure 5, the tip and root vortices' locations in the measurement plane are revealed by ridges corresponding to the peaks in Figure 4(b). The breadth of the ridges narrows with increasing $\lambda$. This is a result of the inherent Reynolds number variation with $\lambda$, which affects the circulation shed into the wake (as seen in Figure 2). This in turn affects the vortex properties and roll-up process discussed in Sec. III C. Qualitatively, the root vortices shown in Figure 5 indicate greater spread of vorticity (broader ridge) suggesting that they are less coherent than the tip vortices at an equivalent azimuthal position. The broad vorticity signal is evident soon after formation and will be shown in Sec. III B to be attributable in part to the blade wakes and interaction of the root vortex with the nacelle. This point is further discussed in the full wake measurements in Sec. III B.

The merging of the tip vortices is highlighted by the circles in Figure 5. Downstream of these circles, two of the three initial tip vortices are entangled. This merging process is detailed in Sec. III B. Increasing the tip speed ratio causes vortex merging to occur closer to the rotor plane, supporting the observations of Felli, Camussi, and Felice ${ }^{8}$ and their hypothesis that mutual inductance is the primary breakdown mechanism of the helical vortex system. The remaining third filament merges further downstream, resulting in a single vortex structure. This single low coherence structure is advected downstream at close to the original pitch of the three helical filament system, qualitatively evident downstream of the red circles in Figure 5. This result has been noted in flow visualizations of helicopter tip vortices ${ }^{6}$ and laser doppler velocimetry measurements of marine propeller wakes. ${ }^{8}$ This single structure could potentially impose cyclic loading on downstream structures.

The helical pitch, $h$, as a function of $\lambda$ can be directly observed from Figure 5 for both the tip and root vortices, and is presented as a function of tip speed ratio in Figure 6. The helical pitch reduces 

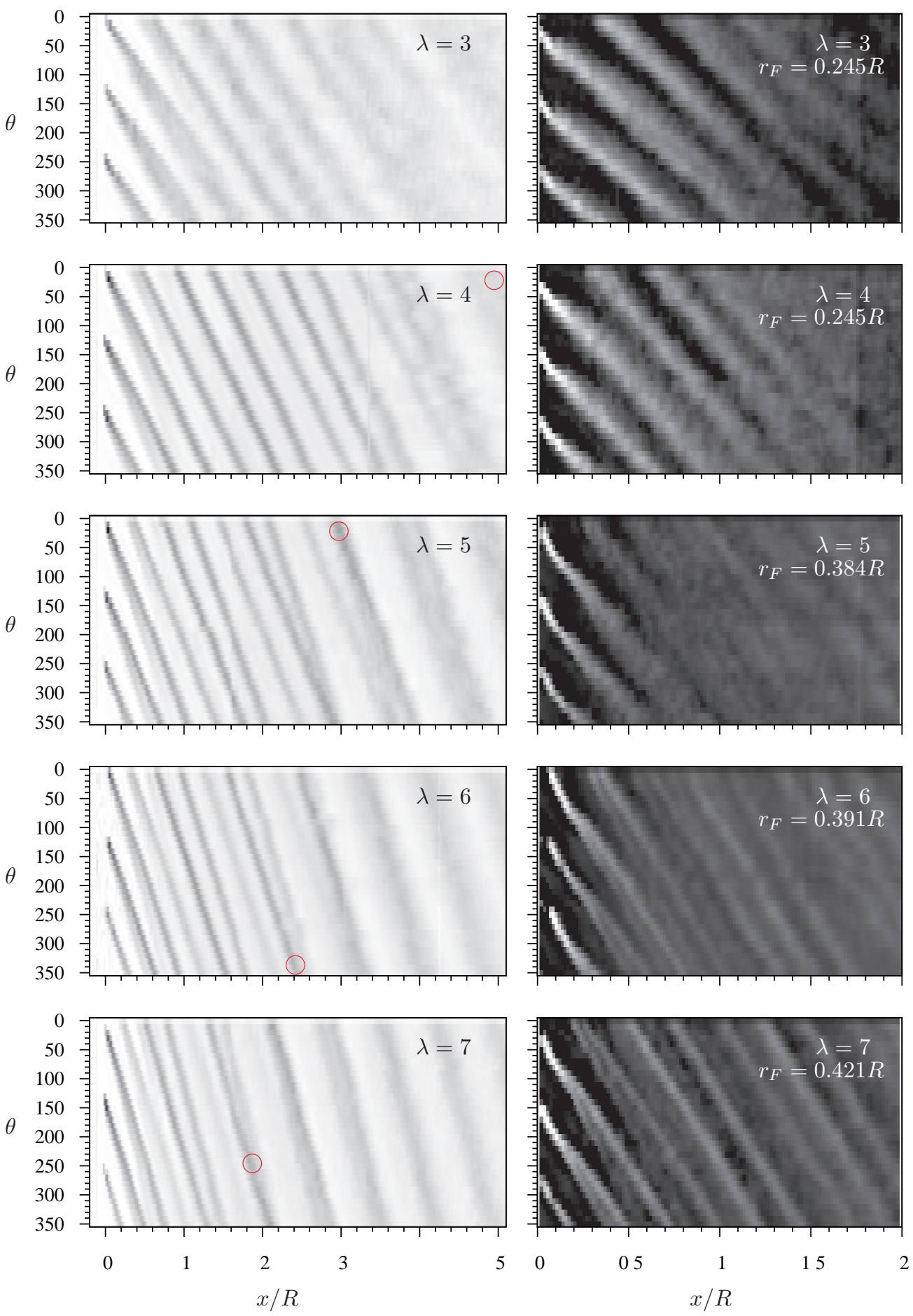

FIG. 5. Streamwise evolution of the phase-locked averaged tip (left) and root (right) vortices. The tip region field of view was constant at $r_{F}=1$ for all $\lambda$, the root vortex field of view center is shown in the figure for each $\lambda$. The circles denote the location where vortices merge, $x_{m} / R$ (see Fig. 10(a)).

with tip speed ratio as vorticity is shed into the wake at a faster rate. The tip vortex helical pitch was determined from the average linear fit of each filament's pitch within the first blade revolution. Due to the rapid deterioration of the root vortex signal, only a third of this range was used to calculate the helical pitch of the root vortices. As $\lambda$ increases, the helical pitch of the root vortices decreases at a 


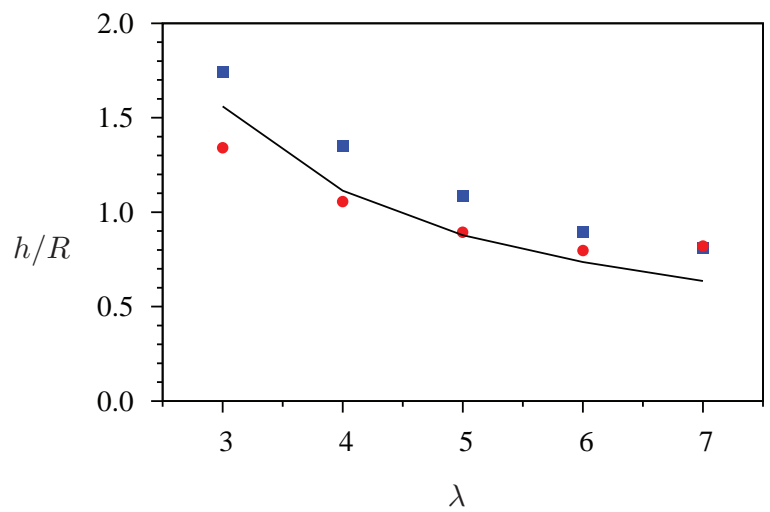

FIG. 6. Average helical pitch of a vortex filament, $h$, for increasing $\lambda$. Squares (blue) denote tip vortex pitch, circles (red) denote root vortices. The solid line represents the predicted tip vortex helical pitch $\left(h_{\mathrm{BEM}}\right)$ based on the BEM methods axial interference factor at the tip.

slower rate than the tip vortices, until they share the same advected speed at $\lambda=7$. The theoretical helical pitch, $h_{\mathrm{BEM}}$, predicted by the BEM method is shown in Figure 6 denoted by the solid line. The predicted helical pitch is calculated ${ }^{21}$ from

$$
h_{\mathrm{BEM}} / R=\frac{2 \pi(1-a)}{\lambda},
$$

where the axial interference factor, $a$, is evaluated by the BEM method at the blade tip for each $\lambda$ and the tangential interference factor is assumed to be negligible. The BEM method helical pitch prediction captures the trend of the tip vortex pitch, however, it is lower than measurements (approximately 10\%-15\% less). This disparity can be attributed in-part to an over-prediction of $a$, due to the $2 \mathrm{D}$ steady assumption of the BEM method.

\section{B. The turbine wake structure}

Section III A highlighted the merging process of the tip and root vortices. However, the Glauert wake model links the tip and root vortices via the blade wakes. Theoretical analysis by Okulov and Sørensen ${ }^{14}$ suggests the presence of the blade wakes affects the stability of the tip vortices. In order to investigate the relationship between the tip and the root vortex evolution, the entire wake structure behind the blade has to be acquired. To this end, wider FOV planar measurements (M2) were taken of the wake at two tip speed ratios: the design condition, $\lambda_{d}=3.5$, and a higher tip speed ratio where the BEM method suggests a smaller blade wake and root vortex formation further away from the nacelle. Referring to Figure 2 , the $\lambda=6$ case was chosen. The data were acquired using phase-locked PIV (at the same spatial resolution as M1) at a single rotor azimuthal angle, $\theta=0^{\circ}$, i.e., where a blade intersected the measurement plane. The measurements captured half of the wake downstream of the rotor up until $x=5 R$.

Figure 7 presents the rotor wake for the design tip speed ratio, $\lambda_{d}$. Freestream flow is from left to right and the blade is rotating in the positive $z$-direction (out of the page). The contours in Fig. 7(a) denote the streamwise velocity, $\bar{U}_{x}$, and Fig. $7(\mathrm{~b})$ the out-of-plane vorticity, $\bar{\omega}_{z}$. From the velocity field, the dominant features of the turbine wake are observable: a wake expansion is made evident by the wake boundary delineated by the unit velocity ratio $\left(\bar{U}_{x} / U_{\infty}=1\right)$ contour line; embedded tip vortices in the wake shear layer; and a velocity deficit in the wake. The velocity deficit is nonuniform across the span of the blade as the lifting surface terminates prior to the rotational axis. The combination of reduced blockage and induced velocity of the root vortex causes a reduction in the velocity deficit in the wake. This is in contrast with recent experiments using the Glauert rotor design, ${ }^{22}$ where the lifting surface extended to the rotational axis.

The vorticity contours in Figure 7(b) reveal the averaged phase-locked signatures of both the tip and root vortex filaments as they intersect the measurement plane. Tip vortices are seen 

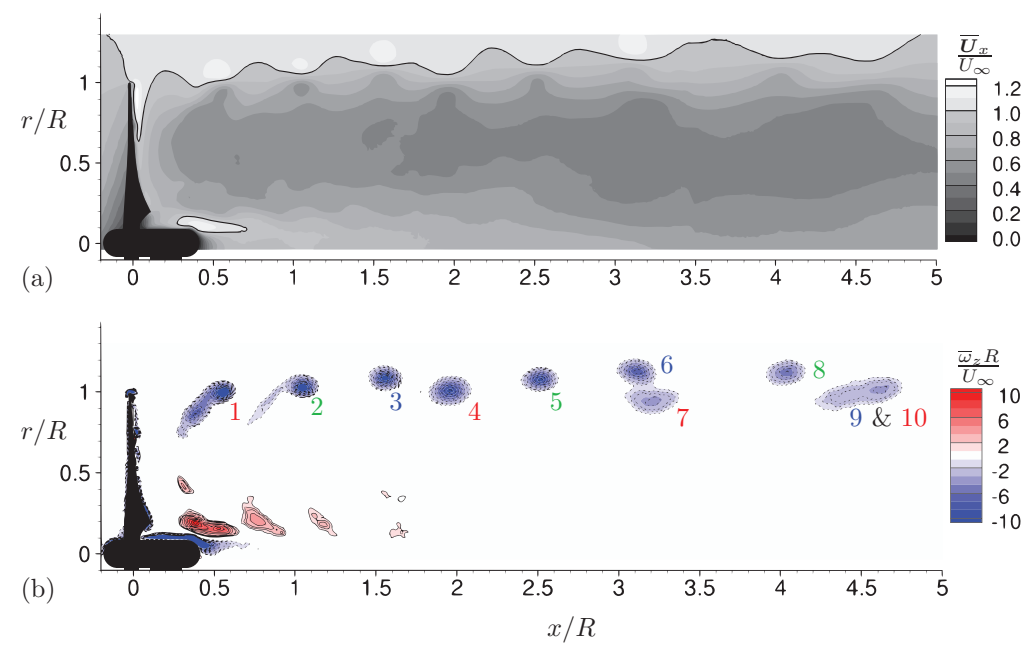

FIG. 7. Phase-locked $\left(\theta=0^{\circ}\right)$ averaged wake structure for $\lambda_{d}=3.5$. Freestream flow is from left to right and the rotor is visible at $x / R=0$. (a) Normalized streamwise velocity field. The solid line denotes $\bar{U}_{x} / U_{\infty}=1$. (b) Normalized vorticity field. Dashed isolines (filled dark grey/blue contours) represent negative vorticity, solid isolines (filled light grey/red contours) represent positive vorticity. Tip and root vortices are present in the wake and the roll-up and pairing of vortices is evident. The tip vortices are numbered sequentially, $n=V_{A} / 120^{\circ}$.

near the tip radial location $(r / R \approx 1.0)$ in clockwise rotation, while the root vortices are inboard $(r / R \approx 0.2)$ in counter-clockwise rotation. The tip vortices are numbered sequentially, with each number representing $n=V_{A} / 120^{\circ}$, where vortex age, $V_{A}$, is the blade travel in degrees. Other features of the wake revealed by the vorticity field include: (i) the roll-up process of the tip and root vortices as they entrain the blade wakes, seen for early vortex ages $(n \leq 3)$; and (ii) a thick shear layer along the nacelle denoted by the negative vorticity on the body.

The merging process revealed in the space-phase diagrams of Figure 5 is apparent in Figure 7(b) as two of the filaments undergo mutual inductance. This mutual induction between filaments can be observed initially in the spacing between the vortices at $n=3$ and 4 . The $n=3$ tip vortex is displaced radially outward and the $n=4$ tip vortex is displaced radially inward due to their co-rotating inductance and both are offset in the axial direction from the ideal helical path. When these two helical vortices next intersect the measurement plane, the mutual inductance has resulted in further displacement of the filaments from the ideal helical path. Here, vortices $n=6$ and 7 occupy approximately the same axial position downstream of the rotor and, through their mutual inductance, tip vortex 6 is moving relatively faster downstream. In the 2D measurement plane, the result of mutual inductance appears similar to the leap-frogging phenomenon seen in vortex rings, ${ }^{34}$ yet due to the helical nature of the filaments, the analogy is limited. The interaction results in vortex merging seen as a single patch of vorticity comprised of the $n=9$ and 10 vortices at $x / R$ $=4.5$. Felli, Camussi, and Felice ${ }^{8}$ interpreted similar results in their experiments as supportive of the hypothesis that mutual inductance is the primary cause of wake breakdown. Our experiments using a three-bladed Glauert turbine operating in the tip speed ratio range $4 \leq \lambda \leq 7$ also support this hypothesis.

Further, the presence of the mutual inductance mode in the phase-locked results suggests it is periodic with rotor phase. This merging process is driven by the Biot-Savart law, which also drives the mutual inductance mode found by Widnall. ${ }^{10}$ In that study, the stability of the mutual inductance mode was found to be a function of vortex strength, core size, and helical pitch. ${ }^{10}$ However, Gupta and Loewy ${ }^{11}$ showed the mutual inductance mode to be the dominant mechanism of helical wake instability regardless of the source of the initial perturbation. The source of the perturbation leading to the saturated state of mutual inductance was hypothesized by Felli et $a .^{35}$ to be the interaction of the blade wake with the subsequent tip vortex. The current results in Figure 7 show a qualitative correlation between the streamwise location of the initial symmetry breaking and the cessation of the roll-up process, denoted by the blade wake vorticity. The curvature of the blade wakes connected 
to the tip vortex increases with wake distance as the vortices on the wake boundary advect faster than the blade wakes in the retarded wake flow (seen in Figure 7). In an analogous manner, the tip vortices of a marine propeller ${ }^{8}$ become unstable first due to their location on the slower wake boundary. Blade wakes have been postulated as a mechanism leading to instability in marine propeller wakes. ${ }^{35}$ While the first evidence of the merging process is analogous, the influence of the blade wake in the two systems are not identical given the inverted wake velocity profiles.

Helical root vortices are also clearly captured behind the rotor visible up to $V_{A}=360^{\circ}$. The root vortex signature located at $r / R \sim 0.2$, ceases closer to the rotor plane compared to the tip vortices, despite the BEM method prediction of larger circulation in the root region for $\lambda_{d}$, see Figure 2 . This is partly attributable to the 2D steady BEM method prediction, which does not account for the centrifugal pumping of the flow along the blades towards the blade tip. This centrifugal pumping causes a radial flow resulting in the tip vortex rather than the root vortex entrainment capturing more of the blade wake vorticity during the roll-up process in the very near wake. The resulting root vortices are therefore likely to be weaker than predicted by the BEM method. Further, the radial location of the root vortex formation at $\lambda_{d}$ causes it to interact with the nacelle boundary layer. This results in the streamwise velocity speed-up in the root region seen in Figure 7 and accounts for the axial stretching of the root vortex profile. The interaction has been shown previously ${ }^{21}$ to play a role in destabilizing the root vortex, and hastens the breakdown of the root vortices.

The wake of the Glauert rotor at the higher tip speed ratio of $\lambda=6$ is shown in the streamwise velocity and vorticity fields of Figure 8 . The velocity contours in Figure 8(a) indicate a lower velocity deficit in the wake compared to $\lambda_{d}$. This is evident particularly close to the rotational axis, signifying that less energy is extracted from the flow by the rotor. At $\lambda=6$, the wake can be categorized broadly into an external tube of low velocity flow, and an internal region of higher velocity. However, the rotor is still operating in a turbine state, with a net energy transfer from the fluid to the structure.

Wake expansion as shown by the streamwise velocity contour line $\overline{U_{x}} / U_{\infty}=1$, is slightly reduced from the $\lambda_{d}$ case as less energy is extracted by the rotor. The velocity gradients indicate the root vortices are displaced radially outward.

The vorticity field in Figure 8(b) reveals the root vortex radial location at $r=0.4 R$, matching the location of the BEM method prediction's large drop in bound circulation shown in Figure 2. At this location, the root vortices contribute to the local velocity speed-up in the internal region due to their counter-clockwise rotation. Blade wakes are captured for $V_{A}<360^{\circ}$, as is the shear layer on the nacelle. Qualitatively, the vorticity field shows very little of the axial stretching of the root vortices

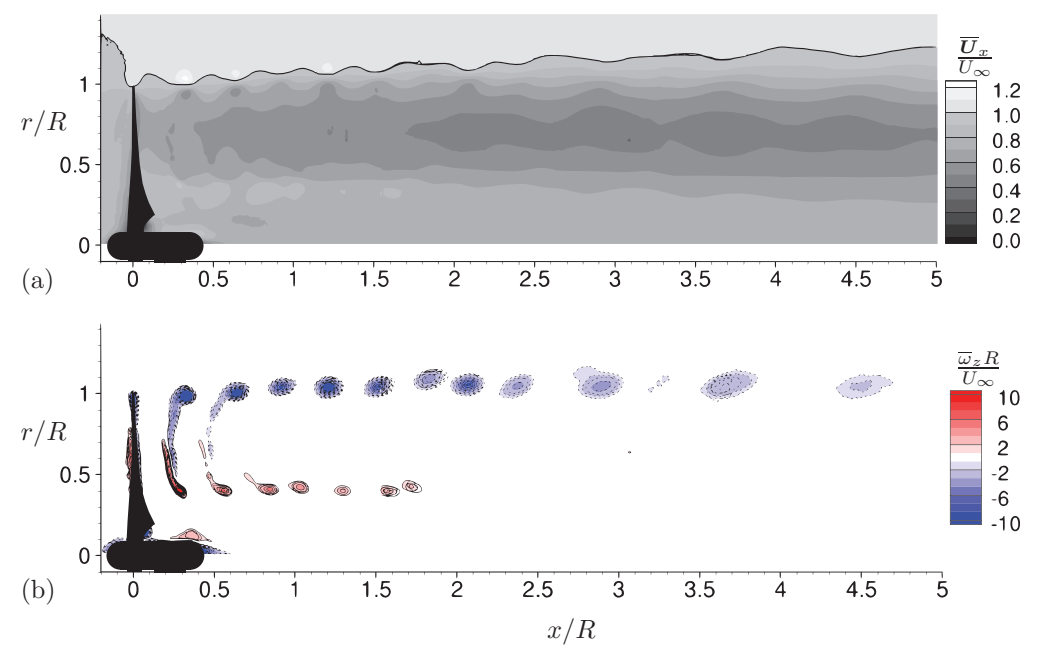

FIG. 8. Phase-locked $\left(\theta=0^{\circ}\right)$ averaged wake structure for $\lambda=6$. Freestream flow is from left to right and the rotor is visible at $x / R=0$. (a) Normalized streamwise velocity field. The solid line denotes $\bar{U}_{x} / U_{\infty}=1$. (b) Normalized vorticity field. Dashed isolines (filled dark grey/blue contours) represent negative vorticity, solid isolines (filled light grey/red contours) represent positive vorticity. 
seen at $\lambda_{d}$, as their radial position further outward reduces interaction with the nacelle shear layer. Moreover, the tip and root vortices have smaller vortex cores compared to the $\lambda_{d}$ case. Similar to the $\lambda_{d}$ case, the tip vortices undergo a mutual inductance, initially observed by their radial displacement from the idealised helical path at $x / R \approx 2$. Vortex merging occurs by $x / R \approx 3$, with a single patch of vorticity advected downstream beyond this location.

The root vortex signatures also demonstrate a mutual inductance between filaments that is not evident in the lower $\lambda_{d}$ results of Figure 7(b). The mutual inductance occurs at an earlier position in the wake, $x / R \sim 1.6$, compared to $x / R \sim 2$ for the tip vortices, as they are embedded in a larger velocity deficit. Again, this is analogous to the observations of Felli, Camussi, and Felice. ${ }^{8}$ However, they found that the hub vortex destabilization followed destabilization of the tip vortices which were embedded in the slower moving wake boundary fluid. ${ }^{8}$

\section{Properties of the helical vortices}

Study of helical vortex evolution in experimental PIV data requires an accurate identification of the vortices in the obtained vector fields. The identification of a vortex is a non-trivial process, in part by the range of definitions and theoretical models available. ${ }^{36}$ The identification of vortices in experimental instantaneous PIV data is further complicated by the inherent error introduced by the PIV technique. ${ }^{37}$ This PIV uncertainty is also exacerbated by the presence of turbulent fluctuations in wake flows, generating high localized vorticity gradients. There have been numerous vortex identification schemes developed, with studies often revisiting the definition of a vortex. ${ }^{36}$

The scheme used in this investigation was outlined by Graftieaux, Michard, and Grosjean. ${ }^{38}$ It exploits the flow topology to identify vortical structures in turbulent flows based on length-scales. The method calculates a scalar, $\Gamma_{1}(x, y)$, at each PIV grid point constrained to a definable interrogation window. This scalar represents the relative rotation about the grid point of the velocity field in the interrogation window. The choice of the window size is dependent on the length-scales of the vortices and background flow length-scales. The value of $\left|\Gamma_{1}\right|$ is bounded by 1 , with $\left|\Gamma_{1}\right|=1$ representing the core center of an axi-symmetric vortex. As the function is not Galilean invariant, the instantaneous Lagrangian flow field surrounding each grid point was estimated by subtracting the phase-locked average velocity from each snapshot. The centers of the vortex cores are identified by calculating the barycenter of the $\Gamma_{1}$ field around the vortex. The vortex core size was determined using a related Galilean invariant scalar field, $\Gamma_{2}(x, y)$, that subtracts the mean velocity in the interrogation window. The function $\Gamma_{2}$ approximates a rotation dominated vector field with a threshold $\Gamma_{2 c r}=2 / \pi$, representing the core boundary of a vortex. ${ }^{38}$

Figure 9 exemplifies typical tip vortex velocity fields captured by PIV, overlaid with the $\Gamma_{2}$ prediction of the vortex core size. Two different vortex ages are presented, $V_{A}=360^{\circ}$ in Figures 9 (a) and 9 (b) and $V_{A}=480^{\circ}$ in Figures 9 (c) and $9($ d), to highlight different stages of the roll-up process. An interrogation window of size $9 \times 9$ vectors was used to extract the vortex radius from the phase-locked average (left of Figure 9) and instantaneous (right of Figure 9) PIV fields. The vertical velocity profile along a horizontal path passing nearest the vortex center is denoted by square symbols. The plots indicate the robustness of the $\Gamma_{2}$ criterion in both phase-locked average and instantaneous velocity fields.

Figure 10 illustrates the variation of the mutual inductance mode onset and merging process with tip speed ratio. Using the $\Gamma_{1}$ criterion for the vortex cores, the location where two vortices shared the same axial position, $x_{m}$, was extracted from the M1 PIV fields at each $\lambda$. Non-dimensionalizing $x_{m}$ by the helical pitch, $h$, the merging occurs at approximately the same $x / h$ for $\lambda>4$. The vortex merging could not be determined for $\lambda<4$ due to the low coherence of the tip vortices. Figure 10(b) quantifies the helical distance behind the rotor where the tip vortices break their initial helical symmetry. This is defined as the location where the $\Gamma_{1}$ derived vortex core locations first deviate from $h$, by more than the arbitrary tolerance, $\Delta x / h \geq 0.01$. There is a very good collapse of the deviation point at $x / h=0.6$. This suggests that the mutual inductance occurs at the same non-dimensional filament length.

Unsteady behavior of vortices is well known: rectilinear trailing vortices ${ }^{39}$ undergo unsteady meander, and helical vortices have also been shown to display aperiodic behavior prior to breakdown. ${ }^{4,21}$ 


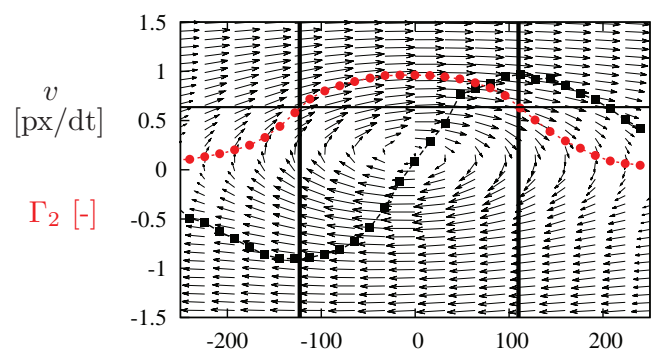

(a)

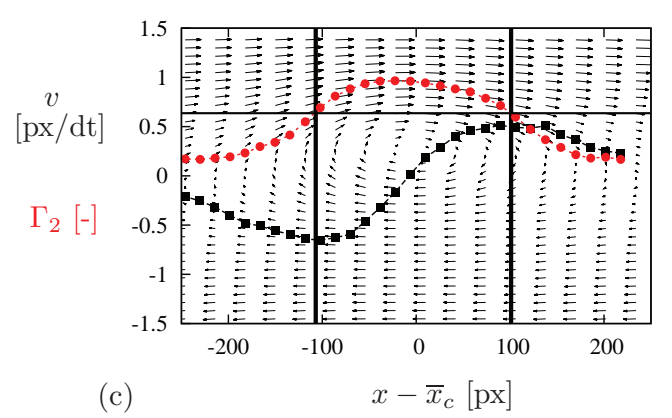

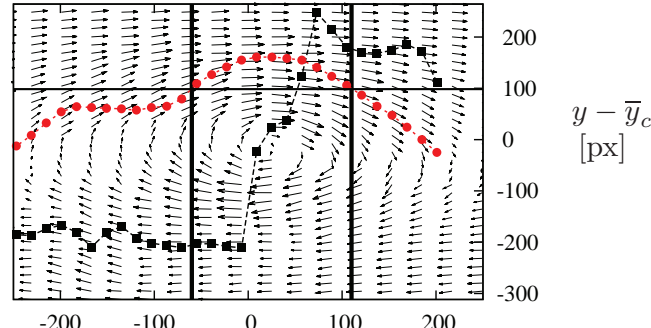

(b)

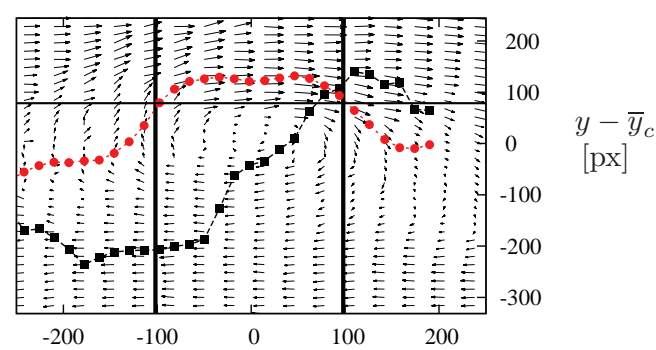

(d)

FIG. 9. Typical phase-locked PIV velocity fields (left: average, right: instantaneous) of the tip vortices, at two tip vortex ages, (a) and (b) is at $V_{A}=360^{\circ}$; (c) and (d) is at $V_{A}=480^{\circ}$. Overlaid are the $\Gamma_{2}$ (red circles) and vertical velocity, $v$, (squares) profiles along horizontal grid points intersecting the vortex center, $\bar{y}_{c}$. The vertical lines denote the vortex core boundary delineated by the threshold, $\Gamma_{2 c r}=2 / \pi$ (horizontal line).

Vortex meander masks the characteristics of trailing vortices in averaged data ${ }^{40}$ due to spatial smoothing. This is also true for averaged phase-locked data which obfuscate non-periodic time-dependent behavior of the vortices. This time-dependent behavior is especially relevant in rotor wakes where the symmetry of the multiple-helical vortex wake is highly sensitive to perturbations..$^{10}$ If the symmetry breakdown along the filament is aperiodic with respect to the rotor phase, it cannot be accurately inferred from a straight application of phase-locked averaging. Therefore, any characterization of the vortex properties, such as vortex strength and core size should account for meander, requiring analysis of instantaneous velocity fields.

To investigate the meander of the vortices in the $\mathbf{M} 2$ measurements, the data were filtered using proper orthogonal decomposition (POD). POD is a flexible mathematical tool used to approximate a data-set using a finite sum of orthogonal basis functions, providing low-rank models of complicated multi-degree-of-freedom dynamic systems, such as fluid flows. ${ }^{41}$ The snapshot method of $\mathrm{POD}^{42}$ is a

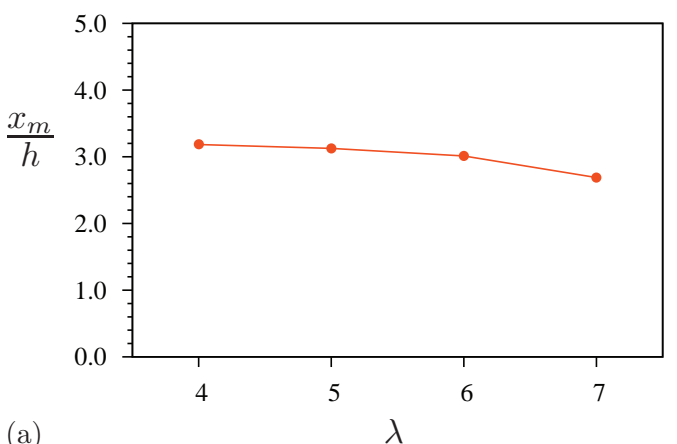

(a)

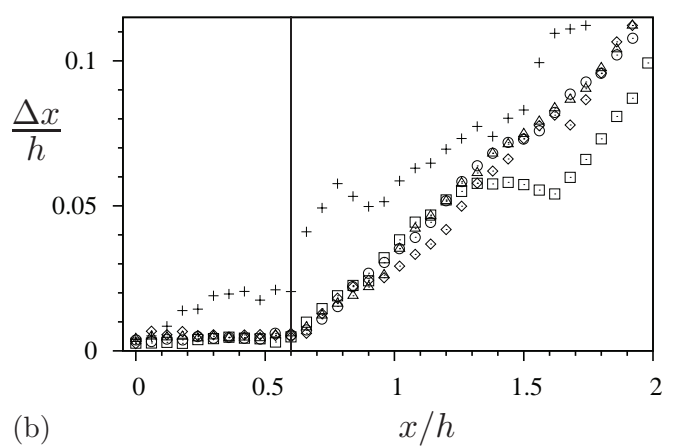

FIG. 10. Variation of the mutual inductance process with tip speed ratio. (a) Location of the onset of vortex merging, $x_{m} / h$, for $4<\lambda<7$, as shown by the red circles in Figure 5. (b) Deviation of the vortices from the extracted helical pitch, $h$, for 3 $<\lambda<7$. Symbols denote tip speed ratio: $\lambda=3$ (crosses), 4 (squares), 5 (circles), 6 (triangles), 7 (diamonds). The departure of the tip vortex from the helical path with pitch, $h$, is designated by the vertical line. 
low-cost algorithm for calculating these basis functions from measured state data. The shape of these basis functions, or modes, may be used to identify correlations across the measured states (mode shapes spanning the state dimensions) or to reconstruct the dynamics of the flow with low-order models.

When considering experimental PIV data, the measured states are velocity vector fields (or another derived variable) spanning the PIV frame comprising $n_{x} \times n_{y}$ grid points. Calculation of the POD modes using the snapshot method begins with the construction of a matrix, $\boldsymbol{A}$, where each column is an instance in time and the rows contain the field values, resulting in $M$ rows and $N$ columns, where $M$ is the number of measured points in the PIV frame, and $N$ is the number of snapshots taken in time. For velocity measurements from planar PIV, the row length is equal to $M=2\left(n_{x} \times n_{y}\right)$. The normalized auto-correlation of the observed snapshots is

$$
\boldsymbol{C}=\frac{1}{N}(\boldsymbol{A}, \boldsymbol{A}) .
$$

A singular value decomposition of $\boldsymbol{C}$ generates $N$ temporal, $\boldsymbol{t}$, and state modes, $\boldsymbol{v}$, and their singular values. The inherent benefit of the above method (as compared to an eigenvalue decomposition or a direct singular value decomposition of $\boldsymbol{A}$ ) is that the singular values are ranked by magnitude and their units are the same as the auto-correlation matrix, $\boldsymbol{C}$. For velocity vector data, this has the same units as the kinetic energy of the flow. These properties of the technique allow for physical interpretations of the related spatial mode shapes and their kinetic energy contribution to the fluid flow. These modes, $\boldsymbol{\Phi}^{(i)}$, are the projections of the state modes, $\boldsymbol{v}_{i}$, of the decomposition onto the measured states, $A^{(i)}$. The spatial modes then allow the reconstruction of the measured states at a time instant,

$$
\boldsymbol{U}^{(i)}=\sum_{j=1}^{N} a_{i j} \boldsymbol{\Phi}^{(j)},
$$

where the coefficients, $a_{i j}$, are the projections of the measured velocity onto the orthogonal basis,

$$
a_{i, j}=\left(\boldsymbol{A}^{(i)}, \boldsymbol{\Phi}^{(j)}\right) .
$$

In order to extract the instantaneous vortex positions, the $\Gamma_{1}, \Gamma_{2}$ fields were first computed using the full phase-locked averaged vector fields. Knowledge of the phase-locked average vortex core locations were exploited to crop the data fields around individual vortices or merged vortices. These fields were then adjusted to the frame of reference of the vortex and the $\Gamma_{1}$ and $\Gamma_{2}$ fields were recomputed on the low-rank POD reconstructed instantaneous fields. The filtering requires a criterion for the number of POD modes to use in the reconstruction. The threshold defined by Epps and Techet ${ }^{43}$ was applied to reconstruct the velocity fields to filter for length scales below that of the PIV methods inherent error $(\epsilon=0.1 \mathrm{px} / \Delta t)$. The filtered instantaneous snapshots were composed of between 10 and 20 PIV modes, containing over $90 \%$ of the kinetic energy.

Figure 11 indicates the instantaneous positions of the tip and root vortices for $\lambda=6$. The distribution of the vortex positions shows that the magnitude of the meander increases with vortex age. This increase in meander highlights an unsteadiness in the evolution of the filaments and is consistent with prior observations ${ }^{4,8,21}$ of additional wavelengths occurring along the helical filaments.

The PIV data also permit vortex properties such as vortex core circulation strength, $\Gamma$, and vortex radius, $r_{c}$, to be extracted from each of the instantaneous frames. This was computed on the M1 measurement set, which had insufficient snapshots to gain from filtering with POD. Vortex core circulation is evaluated by a line integral around the $\Gamma_{2 c r}$ contour after the vortex roll-up process is complete (i.e., $V_{A}=360^{\circ}$ ),

$$
\Gamma=\oint_{\Gamma_{2 c r}} \boldsymbol{u} \cdot \boldsymbol{d} \boldsymbol{l} .
$$

Figure 12 presents the tip vortex core circulation and core radius at $V_{A}=360^{\circ}$ for both phaselocked instantaneous and averaged fields. There is good agreement between the phase-locked averaged data and the average of the instantaneous phase-locked data fields. This suggests the spatial 


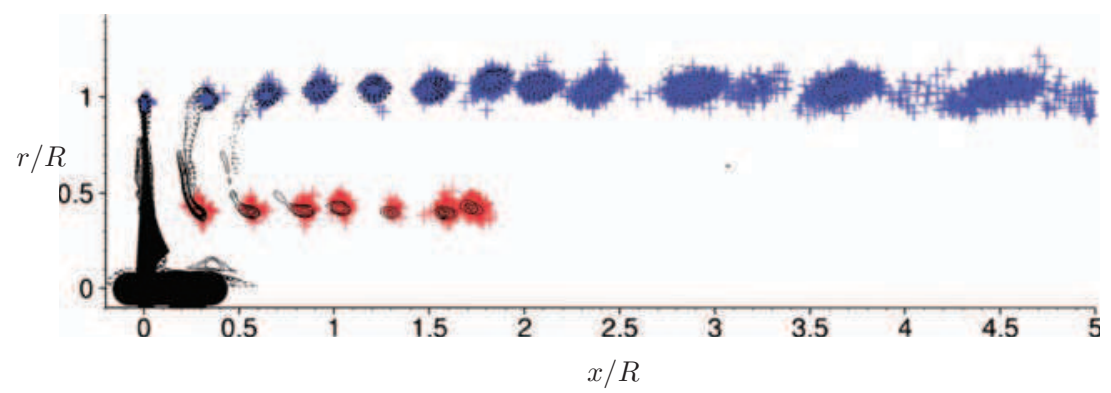

FIG. 11. Position of the instantaneous vortex cores for $\lambda=6$, represented by crosses for the tip (dark grey/blue) and root (light grey/red) helical vortices. Meander of the vortex cores visibly increases with age of the vortex.

smoothing effect of vortex meander is minimal at the early vortex age of $V_{A}=360^{\circ}$ for most tip speed ratios. In support of this hypothesis, meander was shown to be minimal at $V_{A}=360^{\circ}$ for $\lambda=6$ in Figure 11 .

Assuming minimal variation of airfoil performance across the operating Reynolds number range, the tip vortex core's circulation reduces with $\lambda$ due to the reduction in blade angle of attack. The results of a lifting line method using the BEM method data of Figure 2 are also presented in Figure 12(a). The lifting line results are determined by summing the radial bound circulation gradient between the maximum blade bound circulation, $\Gamma_{B, \max }(r)$ position and the tip as shown in Eq. (7)

$$
\Gamma=\kappa\left(-\Gamma_{B}(R)+\int_{r_{\max }}^{R} \frac{\mathrm{d} \Gamma_{B}}{\mathrm{~d} r} \mathrm{~d} r\right)=-\kappa \Gamma_{B}\left(r_{\max }\right),
$$

where $r_{\text {max }}$ denotes the radial location of maximum bound circulation, and $\kappa$ is a determinable coefficient accounting for the distribution of vorticity contained in the core and dependent upon vortex profile, Reynolds number, and the 2D assumptions of the BEM method. The vorticity shed into the wake is equivalent to the maximum blade bound circulation, $\Gamma_{B, \max }$. Betz's formation theory of trailing vortices ${ }^{44}$ states $\Gamma_{B, \max }$ should be contained in the fully developed tip vortices. Numerical simulations of wind turbine wakes have shown that the tip vortices contain approximately $80 \%$ of $\Gamma_{B, \max }{ }^{45}$ However, the core of the helical tip vortices contains even less. ${ }^{15,46}$ Experimental measurements ${ }^{47}$ have shown that the helical tip vortex cores of a two-bladed helicopter rotor initially contained only $70 \%$ of $\Gamma_{B, \max }$, i.e., $\Gamma / \Gamma_{B, \max }=0.7$, and by $V_{A}=120^{\circ}$, reduced to $\Gamma / \Gamma_{B, \max }$ $\sim 0.58$. Vortex models including Iversen's and Tung's, and Ramasamy's transitional tip vortex model predict the core to contain between $33 \%$ and $74 \%$ of the tip vortex circulation. ${ }^{46}$ Our current
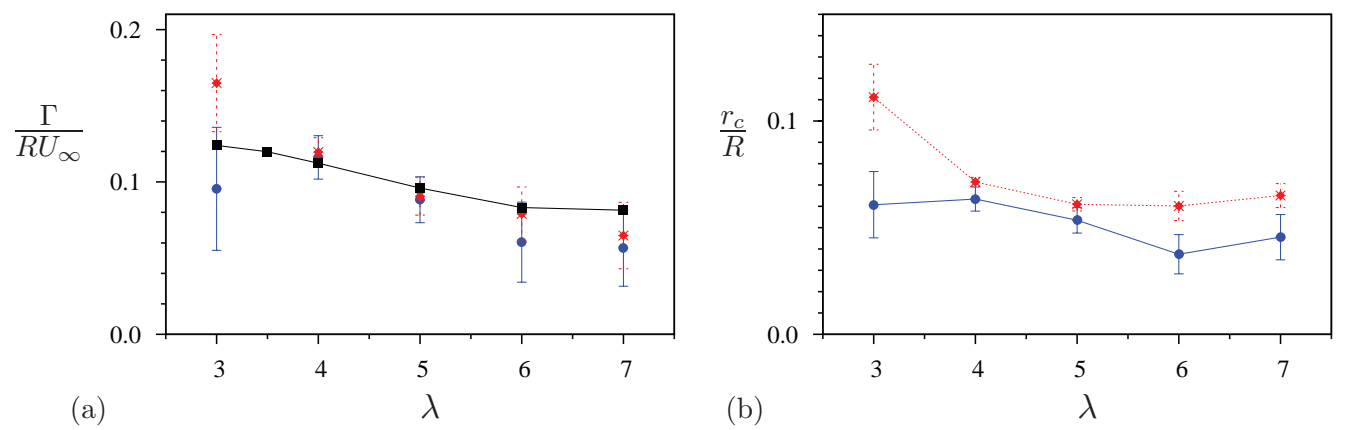

FIG. 12. (a) Circulation strength of the tip vortex cores, $\Gamma / R U_{\infty}$, after the vortex roll-up process $\left(V_{A}=360^{\circ}\right)$ is complete for increasing $\lambda$. Circles (blue) represent the mean value of instantaneous vortex properties, whereas the diamonds (red) denote properties of the phase-locked averaged vortices. Filled black squares represent $50 \%$ of the maximum blade bound circulation using the BEM method data from Figure 2. (b) Radius of the tip vortex cores, $r_{c} / R$, at $V_{A}=360^{\circ}$ with increasing tip speed ratio. 
results show that $\kappa=0.5$ provides a good agreement between the measured tip vortex core circulation and the BEM method prediction.

The vortex core radius is denoted as a circle of equivalent area to the $\Gamma_{2 c r}$ contour and is shown on the right of Figure 12. Vortex core radius is dependent on $\lambda^{26}$ due to the angle of attack, $\alpha$, reduction in the tip region. The BEM method results indicate the tip angle of attack, $\alpha(R)$, ranges between $0.82^{\circ} \leq \alpha(R) \leq 8.7^{\circ}$ for the $\lambda$ range shown in Figure 12. The Glauert rotor was designed to operate near the upper limit of the attached region of airfoil performance at $\lambda_{d}$. Therefore at $\lambda$ $<\lambda_{d}$, laminar separation is likely, causing time-dependent airfoil performance, leading to the large standard deviation at $\lambda=3$.

\section{CONCLUSIONS}

In this study, we have investigated the wake structure of an optimized Glauert rotor. The results underline the importance of blade design for experimental studies and the influence blade aerodynamics have on the wake structure. For high $\lambda$, the results revealed a blade loading that resulted in root vortices forming at a sufficient radial distance from the nacelle reducing interaction with the nacelle boundary layer. This has a strong influence on the root vortex formation, as well as the blade wakes and likely interaction dynamics between the tip and root vortices.

The experiments captured the tip vortex mutual inductance leading to a merging of the vortices, in-line with prior helical vortex investigations. ${ }^{6,8}$ The first evidence of mutual inductance, determined by the displacement of a tip or root vortex from its idealized path, coincided at the same nondimensionalized distance, $x_{m} / h$, for all $\lambda$. The previously undocumented root vortex evolution and mutual inductance was also captured. The mutual inductance of the root vortices occurred at an earlier axial position compared to the tip vortices, analogous to observations of a $N+1$ vortex system by Felli, Camussi, and Felice. ${ }^{8}$ The meander of the tip and root vortex positions in phaselocked results represents an aperiodicity with blade angle and is consistent with the hypothesis ${ }^{10}$ and observations ${ }^{8}$ of multiple wavelengths present along the helical vortex filaments.

Similar to marine propellers ${ }^{8}$ and helicopter rotors, ${ }^{6}$ vortex merging results in a re-organization of the HAWT wake. Post vortex merging, patches of vorticity with low coherence remain in the wake. Thus, the far-wake of a wind turbine may contain weak but periodic helical vortex structures requiring consideration for wind farm planning.

\section{ACKNOWLEDGMENTS}

M.S. would like to thank the group at DTU Wind Energy for the many fruitful discussions during his stay in 2010. The authors acknowledge the financial support of the Australian Research Council through the ARC Discovery Project DP1096444.

${ }^{1}$ P. A. Alfredsson and J. A. Dahlberg, "A preliminary wind tunnel study of windmill wake dispersion in various flow conditions," Technical Report No. aU-1499 (The Aeronautical Research Institute of Sweden, 1979), Part 7.

${ }^{2}$ J. H. Walther, M. Guénot, E. Machefaux, J. T. Rasmussen, P. Chatelain, V. L. Okulov, J. N. Sørensen, M. Bergdorf, and P. Koumoutsakos, "A numerical study of the stability of helical vortices using vortex methods," J. Phys.: Conf. Ser. 75, 012034 (2007).

${ }^{3}$ S. Ivanell, R. Mikkelsen, J. N. Sørensen, and D. Henningson, "Stability analysis of the tip vortices of a wind turbine," Wind Energy 13, 705-715 (2010).

${ }^{4}$ I. Dobrev, B. Maalouf, N. Troldborg, and F. Massouh, "Investigation of the wind turbine vortex structure," in Proceedings of the 14th International Symposium on Applications of Laser Techniques to Fluid Mechanics, Lisbon, Portugal, 7-10 July 2008.

${ }^{5}$ H. Hu, Z. Yang, and P. Sarkar, "Dynamic wind loads and wake characteristics of a wind turbine model in an atmospheric boundary layer wind," Exp. Fluids 52, 1277-1294 (2012).

${ }^{6}$ F. Caradonna, "Performance measurement and wake characteristics of a model rotor in axial flight," J. Am. Helicopter Soc. 44, 101-108 (1999).

${ }^{7}$ M. J. Bhagwat and J. G. Leishman, "Stability analysis of helicopter rotor wakes in axial flight," J. Am. Helicopter Soc. 45, 165-178 (2000).

${ }^{8}$ M. Felli, R. Camussi, and F. D. Felice, "Mechanisms of evolution of the propeller wake in the transition and far fields," J. Fluid Mech. 682, 5-53 (2011).

${ }^{9}$ H. Levy and G. Forsdyke, “The steady motion and stability of a helical vortex,” Proc. R. Soc. London, Ser. A 120, 670-690 (1928). 
${ }^{10}$ S. Widnall, “The stability of a helical vortex filament,” J. Fluid Mech. 54, 641-663 (1972).

${ }^{11}$ B. P. Gupta and R. G. Loewy, "Theoretical analysis of the aerodynamic stability of multiple, interdigitated helical vortices," AIAA J. 12, 1381-1387 (1974).

${ }^{12}$ D. H. Wood and J. Boersma, "On the motion of multiple helical vortices," J. Fluid Mech. 447, 149-171 (2001).

${ }^{13}$ V. L. Okulov, "On the stability of multiple helical vortices," J. Fluid Mech. 521, 319-342 (2004).

${ }^{14}$ V. L. Okulov and J. N. Sørensen, "Stability of helical tip vortices in a rotor far wake," J. Fluid Mech. 576, 1-25 (2007).

${ }^{15} \mathrm{~K}$. W. McAlister and J. T. Heineck, "Measurements of the early development of trailing vorticity from a rotor," Technical Report No. TP-2002-211848 (National Aeronautics and Space Administration, 2002).

${ }^{16}$ R. J. Barthelmie, K. Hansen, S. T. Frandsen, O. Rathmann, J. G. Schepers, W. Schlez, J. Phillips, K. Rados, A. Zervos, E. S. Politis, and P. K. Chaviaropoulos, "Modelling and measuring flow and wind turbine wakes in large wind farms," Wind Energy 12, 431-444 (2009).

${ }^{17}$ Y. Hattori and Y. Fukumoto, "Short-wavelength stability analysis of a helical vortex tube," Phys. Fluids 21, 014104 (2009).

${ }^{18}$ L. J. Vermeer, "A review of wind turbine wake research at TU Delft," A Collection of the 2001 ASME Wind Energy Symposium Technical Papers (ASME, New York, 2001), pp. 103-113.

${ }^{19}$ J. N. Sørensen, "Aerodynamic aspects of wind energy conversion," Annu. Rev. Fluid Mech. 43, 427-428 (2011).

${ }^{20}$ J. N. Sørensen, "Instability of helical tip vortices in rotor wakes," J. Fluid Mech. 682, 1-4 (2011).

${ }^{21}$ M. Sherry, J. Sheridan, and D. Lo Jacono, "Characterisation of a horizontal axis wind turbine's tip and root vortices," Exp. Fluids 54, 1-19 (2013).

${ }^{22}$ I. V. Naumov, V. V. Rahmanov, V. L. Okulov, C. M. Velte, K. E. Meyer, and R. F. Mikkelsen, "Flow diagnostics downstream of a tribladed rotor model," Thermophys. Aeromechanics 19, 171-181 (2012).

${ }^{23}$ H. Glauert, in Airplane Propellers, Aerodynamic Theory, edited by W. F. Durand (Dover, New York, 1935), pp. 169-360.

${ }^{24}$ E. N. Jacobs and A. Sherman, "Airfoil section characteristics as affected by variations of the Reynolds number," Technical Report No. 586 (National Advisory Committee for Aeronautics, 1937).

${ }^{25}$ R. E. Wilson and P. B. S. Lissaman, “Applied aerodynamics of wind power machines," Technical Report No. NSF-RA-N74-113 (Oregon State University, 1974).

${ }^{26}$ N. J. Vermeer, "How big is a tip vortex?" (Institute for Wind Energy, Delft University of Technology, 1996), pp. 77-82.

${ }^{27}$ P. R. Ebert and D. H. Wood, "The near wake of a model horizontal-axis wind turbine. Part 2: General features of the three-dimensional flowfield," Renewable Energy 18, 513-534 (1999).

${ }^{28}$ W. Z. Shen, R. M. Mikkelsen, J. N. Sørensen, and C. Bak, "Tip loss corrections for wind turbine computations," Wind Energy 8, 457-475 (2005).

${ }^{29}$ M. Nazarinia, D. Lo Jacono, M. C. Thompson, and J. Sheridan, "Flow behind a cylinder forced by a combination of oscillatory translational and rotational motions," Phys. Fluids 21, 051701 (2009).

${ }^{30} \mathrm{M}$. Sherry, J. Sheridan, and D. Lo Jacono, "An experimental investigation of the recirculation zone formed downstream of a forward facing step,” J. Wind. Eng. Ind. Aerodyn. 98, 888-894 (2010).

${ }^{31}$ D. Tudball-Smith, J. S. Leontini, J. Sheridan, and D. Lo Jacono, "Streamwise forced oscillations of circular and square cylinders," Phys. Fluids 24, 111703 (2012).

${ }^{32}$ A. Nemes, J. Zhao, D. Lo Jacono, and J. Sheridan, "The interaction between flow-induced vibration mechanisms of a square cylinder with varying angles of attack," J. Fluid Mech. 710, 102-130 (2012).

${ }^{33}$ A. Fouras, D. Lo Jacono, and K. Hourigan, "Target free stereo PIV: A novel technique with inherent error estimation and improved accuracy,” Exp. Fluids 44, 317-329 (2008).

${ }^{34}$ T. T. Lim and T. B. Nickels, Fluid Vortices (Kluwer, Boston, 1995), Chap. 4.

${ }^{35}$ M. Felli, F. Di Felice, G. Guj, and R. Camussi, "Analysis of the propeller wake evolution by pressure and velocity phase measurements," Exp. Fluids 41, 441-451 (2006).

${ }^{36}$ P. Chakraborty, S. Balachandar, and R. J. Adrian, "On the relationships between local vortex identification schemes," J. Fluid Mech. 535, 189-214 (2005).

${ }^{37}$ M. Raffel, C. Willert, and J. Kompenhans, Particle Image Velocimetry: A Practical Guide (Springer, New York, 2002).

${ }^{38}$ L. Graftieaux, M. Michard, and N. Grosjean, "Combining PIV, POD and vortex identification algorithms for the study of unsteady turbulent swirling flows," Meas. Sci. Technol. 12, 1422-1429 (2001).

${ }^{39}$ W. J. Devenport, M. C. Rife, S. I. Liapis, and G. J. Follin, “The structure and development of a wing tip vortex," J. Fluid Mech. 312, 67-106 (1996).

${ }^{40}$ A. L. Heyes, R. F. Jones, and D. A. R. Smith, "Wandering of wing-tip vortices," in Proceedings of the 12th International Symposium on the Applications of Laser Techniques to Fluid Mechanics, Lisbon, Portugal, 2004.

${ }^{41}$ G. Berkooz, P. Holmes, and J. L. Lumley, "The proper orthogonal decomposition in the analysis of turbulent flows," Annu. Rev. Fluid Mech. 25, 539-575 (1993).

${ }^{42}$ L. Sirovich, "Turbulence and the dynamics of coherent structures. Part I: Coherent structures,” Q. Appl. Math. 45, 561-571 (1987).

${ }^{43}$ B. P. Epps and A. H. Techet, "An error threshold criterion for singular value decomposition modes extracted from PIV data," Exp. Fluids 48, 355-367 (2010).

${ }^{44}$ A. Betz, "Behaviour of vortex systems," Technical Report No. NACA TM-713 (National Advisory Committee for Aeronautics, 1933).

${ }^{45}$ S. Ivanell, R. Mikkelsen, J. N. Sørensen, and D. Henningson, “Analysis of numerically generated wake structures," Wind Energy 12, 63-80 (2009).

${ }^{46}$ M. Ramasamy and J. Leishman, "Reynolds number-based blade tip vortex model," in Proceedings of the 61st Annual Forum and Technology Display of the American Helicopter Society International (AHS International, 2007), Vol. 61, pp. 1-13.

${ }^{47}$ M. J. Bhagwat and J. G. Leishman, "Measurements of bound and wake circulation on a helicopter rotor," J. Aircraft 37(2), $227-234(2000)$. 